Kelly, B. K., Miller, G. A. \& Hale C. W. (1952). J. gen. Microbiol. 6, 41-46.

\title{
Gulture Media for Large-scale Production of Micrococcin
}

\author{
By B. K. KELLY, G. A. MILLER AND C. W. HALE \\ Antibiotics Research Station (Medical Research Council), Clevedon, Somerset
}

\begin{abstract}
SUMMARY: A number of media have been developed for the production of micrococcin. A satisfactory medium consists of corn steep liquor to $1800 \mathrm{mg}$. N/l. with an aqueous extract of dried autolysed yeast to $1800 \mathrm{mg}$. N/1., which gives titres of the order of $40-60 \mu \mathrm{g}$. micrococin $/ \mathrm{ml}$. in 3 days. A soluble yeast extract such as Marmite may be used in place of the dried autolysed yeast. Moderate aeration is required for good titres but initial $\mathrm{pH}$ is relatively unimportant, any value in the range $6 \cdot 0-7 \cdot 0$ being suitable. The main energy source in sugar-free media is probably the aerobic oxidative deamination of amino-acids. An inoculum that has been pre-incubated gives better results than an inoculum used immediately after it has been prepared. The addition of various inorganic ions to the medium was tried without any detectable influence on titre.
\end{abstract}

The production of micrococcin $(\mathrm{Su}, 1948)$ in quantity was undertaken to provide material with which other workers could examine extraction of the active substance and its behaviour both in vitro and in vivo. The principal object of the present experiments was to reduce the cost of the medium and to supply information necessary for fairly large-scale fermentation. Although a number of ad hoc experiments were carried out in shake flasks, stirred aerated flasks were used for most of the work. The correlation between the results obtained in stirred aerated flasks and those obtained in a 100 gal. stirred aerated fermenter was good.

\section{METHODS}

Stirred aerated flasks. The stirred aerated flask (Fig. 1), designed to study general fermentation problems, has been in use for more than 3 years. During this period a number of fungi have been examined. No difficulties, apart from control of foaming, were found in getting a sufficient supply of oxygen to the fungi examined, some of which had an extremely high oxygen demand.

The basis of the design is a bolt-head Chance Hysil flask of 5 l. capacity, normally containing $4 \mathrm{l}$. of medium. The flask is fitted with a black rubber bung having five moulded holes through which pass the aerators, stirrer, sampling arm and the air outlet filter. The rubber bung is retained in the flask by a suitably perforated metal plate clamped to a metal ring round the neck of the flask by butterfly nuts. The aerator arms, which have a bore of $\frac{1}{4}$ in., are not restricted at the nozzles. They carry baffle plates preventing vortices and increasing the agitation effect. A relatively small stirrer facilitates assembly. It operates above the aerator outlets and disperses the air in the medium. A range of stirring speeds from 100 to 1000 r.p.m. is available. Greater agitation can be obtained by the addition of links of stainless steel chain to the blades of the stirrer, but excessive speeds with such chains have 
been found by Mrs Gill-Carey (private communication) to damage extensively a species of fungus. The stirrer shaft operates through a plain metal bearing which is filled with phenolated vaseline immediately after sterilization. A grease-cup, mounted on the shaft below the bearing, prevents the lubricant entering the medium.

Sterile air, pre-heated to the temperature required and automatically humidified, is passed into the flask via a $Y$-piece, and leaves by the outlet air filter, where its rate of flow is measured on a U-tube flowmeter. Units of four flasks, with an air filter and distributing manifold, are mounted on an aluminium frame which is sterilized with all connexions attached except the drive to the stirrer shafts and those to the air flowmeters. Four such frames are mounted, after sterilization, on a metal rack which carries the flowmeters and an electric motor with pulleys, gears and shafting for stirring. The stirrer shafts are connected by flexible metal cables to the vertical driving shafts. The method of transmitting the drive from the motor can be seen in the photograph of the rack (Pl. 1). The racks run on the monorail system devised by Green (1946) for the penicillin production unit, from which the racks were modified.

Shake flasks. Conical flasks (Pyrex, $200 \mathrm{ml}$.) containing $50 \mathrm{ml}$. medium were used on a horizontal shaker giving 84 oscillations/min., the length of throw being $7.5 \mathrm{~cm}$.

Aeration characteristics of the apparatus employed. A number of determinations of the 'aeration constant' (Wise, 1951) for the stirred flasks under conditions normally used were carried out by Mr W. S. Wise. From the results and from experiments on the effect of stirring and aeration, it was clear that the aeration constant necessary to satisfy the peak oxygen demand of the micrococcus used was less than $\mathbf{1} \cdot \mathbf{0}$.

Organism. A strain of Micrococcus $\mathrm{sp}$. isolated by $\mathrm{Su}$ (1948) was used. This strain is now available from the National Collection of Type Cultures (NCTC 7128).

Assay. The method used to assay fermentation samples was the solid medium dilution method of Su (1948), as modified by Heatley, Kelly \& Smith (1952). It is a time-consuming technique, and the number of assay samples was kept to a minimum by pooling equal volumes of samples from replicate fermentations before assay. Arbitrary times for sampling were selected after preliminary observations on the course of the development of antibiotic activity.

Conditions used in comparison of media. Fermentations were carried out at $30^{\circ}$ and the time of incubation was 4 days. Each flask was stirred at 300 r.p.m. and aerated at $3 \mathrm{l}$. air $/ \mathrm{min} . / 4 \mathrm{l}$. medium. The inoculum was $15 \mathrm{ml}$. of a $17 \mathrm{hr}$. stirred aerated culture of the micrococcus grown in nutrient broth at $30^{\circ}$.

Foaming was controlled by the addition of a mixture of D.C. Antifoam 'A' (a Dow Corning silicone product) and tri-amyl citrate. Usual concentrations used were 40 p.p.m. of the former and 100 p.p.m. of the latter, but these were increased when necessary, e.g. with corn steep liquor (CSL), blood preparations 
and casein. No toxicity or diminution in titre was demonstrated when using large amounts of these agents. The antifoam was added as a solution in chloroform before sterilization.

\section{MEDIA}

Su (1948) employed a medium comprising $1 \%$ Lab-Lemco, 1 \% Eupeptone no. 2 (Allen and Hanburys, Ltd.), and $\mathbf{0 . 5} \%$ sodium chloride. It was desirable to find a cheaper and more easily available medium. To this end the materials included in Table 1 were chosen for examination as substitutes for the components of Su's medium. Control runs using Su's medium were always included as a standard of reference for efficiency in micrococcin production. In the first instance, substitutions were based on equal nitrogen contents, but promising combinations were further examined by varying the total nitrogen content and the relative proportions of the two constituents.

Table 1. Source of supply and total nitrogen (TN) of materials tested in media

(Total nitrogen determinations by micro-Kjeldahl.)

$\left.\begin{array}{cc}\text { Material } & \multicolumn{1}{c}{\begin{array}{c}\text { Total } \\ \text { nitrogen } \\ (\%, w / w)\end{array}} \\ \text { Constituents of Su's medium } \\ \text { Oxo Ltd. }\end{array}\right] \begin{gathered}6.98 \\ \text { Allen and Hanburys Ltd. }\end{gathered}$

Lab-Lemco

Substitutes for constituents of Su's medium

$\begin{array}{llr}\text { Corn Steep liquor (CSL) } & \begin{array}{c}\text { Brown and Polson Ltd. (via The Distillers } \\ \text { Co. Ltd.) }\end{array} & \mathbf{2 \cdot 6 0} \\ \text { Dried autolysed yeast (DAY)* } & \text { The Distillers Co. Ltd. } & \mathbf{7 \cdot 1 6} \\ \text { Brewer's yeast } & \text { George's Brewery Ltd., Bristol } & \mathbf{2 \cdot 3 0} \\ \text { Baker's yeast } & \text { The Distillers Co. Ltd. } & \mathbf{2 \cdot 4 0} \\ \text { Guinness yeast extract } & \text { Arthur Guinness, Son \& Co. Ltd. } & \mathbf{5 \cdot 6 0} \\ \text { Yeastrel } & \text { Brewers' Food Supply Co. } & \mathbf{5 \cdot 8 8} \\ \text { Marmite } & \text { The Marmite Food Extract Co. } & \mathbf{6 \cdot 0 4} \\ \text { Meat extract (Argentine) } & \text { Armour and Company Ltd. } & \mathbf{8 \cdot 4 5} \\ \text { Meat meal } & \text { British Glues and Chemicals Ltd. } \\ \text { Maggi's Bouillon powder } & \text { Marber and Co. (Food Products) Ltd. } & \mathbf{9 \cdot 1 5} \\ \text { Bacto-Peptone } & \text { Difco Laboratories Inc. } & \mathbf{4 \cdot 8 0} \\ \text { Fish meal } & \text { Hull Fish Meal and Oil Co. } & \mathbf{1 5 \cdot 5} \\ \text { Casein } & \text { Casein (Industries) Ltd. } & \mathbf{1 3 \cdot 2} \\ \text { Soya flour } & \text { Brodie and Co. Ltd. } & \mathbf{1 2 \cdot 7} \\ \text { Rothes pot ale (concentrated) } & \text { The Distillers Co. Ltd. } & \mathbf{7 \cdot 3}\end{array}$

* Dried autolysed yeast (DAY) extract was made by autoclaving an aqueous suspension (12 kg. DAY/100 l. water) for a short while and then clarifying with a Sharples centrifuge. Other partially soluble materials were acid-hydrolysed before use.

Finally selected medium based on statistical evaluation of the results of test runs. After numerous experiments in stirred or shaken flasks the best medium was found to be CSL to provide $1800 \mathrm{mg} . \mathrm{N} / \mathrm{l}$., DAY extract to provide $1800 \mathrm{mg} . \mathrm{N} / \mathrm{l}$., and $0.5 \%$ sodium chloride. Marmite to the same nitrogen content could replace the DAY extract with only slight decrease of titre and had the advantage of eliminating the DAY extraction step in medium preparation. Some batches of CSL could be employed alone at $3600 \mathrm{mg}$. N/l. but others 
appeared to contain something which was detrimental to micrococcin production at this concentration. All batches of CSL examined gave satisfactory results in combination with DAY extract or Marmite.

Larger scale production. The process resulting from the work here reported was successfully applied to the production of micrococcin in a 100 gal. stainless steel fermenter which was developed from a Kestner stirred and jacketed vessel. Using CSL/Marmite medium, titres of 40-50 $\mu \mathrm{g}$. micrococcin/ ml. were regularly produced in $66 \mathrm{hr}$.; under the same conditions in this apparatus Su's medium gave titres of $6-8 \mu \mathrm{g} . / \mathrm{ml}$.

Comments on various factors. The experiments on media composition showed that any of the materials tried were satisfactory substitutes for the peptone in Su's medium, DAY extract and acid-hydrolysed dried blood in particular giving substantially better results. The best substitute for Lab-Lemco was CSL, but even this gave only slightly better results. CSL gave particularly good titres in combination with acid-hydrolysed dried blood, but this was not pursued further as it proved difficult to recover micrococcin from broths which included blood.

The fermentation is relatively insensitive to variations in conditions. The initial $\mathrm{pH}$ was varied over the range $6 \cdot 0-7 \cdot 4$ without detectable effect. It was found to be advantageous to increase the rate of stirring to 600 r.p.m. with an aeration rate of $\mathbf{3} \mathrm{l}$. air/min., but even under these conditions the aeration constant (Wise, 1951) of the apparatus employed is still less than $\mathbf{1} \cdot \mathbf{0}$.

It was possible to use as inoculum a suspension obtained by washing off $20 \mathrm{oz}$. slants directly, or after only $7 \mathrm{hr}$. incubation, with only a slight drop in yield. Increases in concentration of sodium or chloride ion were made without effect on titre, as were additions of potassium, magnesium, phosphate, and sulphate ion. There was some evidence of a slight drop in micrococcin production with very long sterilization times at $15 \mathrm{lb}$./sq.in. but no difference was observed up to $1 \mathrm{hr}$.

\section{BIOCHEMICAL OBSERVATIONS}

During fermentation in a $\mathbf{1 0 0}$ gal. vessel with CSL/Marmite medium, lactate was rapidly destroyed, acetic acid and ammonia accumulated and the $\mathrm{pH}$ value rose (Fig. 2). The ammonia found was equivalent to about $25 \%$ of the available nitrogen.

By the use of cell suspensions of the micrococcus it was observed that $\alpha$-alanine, glutamic acid and arginine were deaminated with the production of ammonia; histidine, lysine, valine and phenylalanine were not. The deamination of alanine occurred only in presence of oxygen, and the rate of deamination was dependent on the rate of aeration (Fig. 3). This reaction proceeded fastest at about $\mathrm{pH} 6-6 \cdot 5$, decreasing rapidly on either side of this range. No pyruvate was demonstrated after cell suspensions had been aerated in the presence of alanine even when toluene was present (Stephenson, 1949) but, whether toluene was present or not, acetic acid equivalent to about $15 \%$ of the alanine deaminated was formed. Neither pyruvate, lactate, citrate nor glucose influenced the rate of deamination of alanine by washed cell suspen- 
sions. Experiments using gelatine or corn steep protein as substrate indicated that the micrococcus had little proteolytic activity.

Statistical analysis of fermentation experiments in shake flasks showed that alanine could, with advantage, replace part of the CSL nitrogen in CSL/

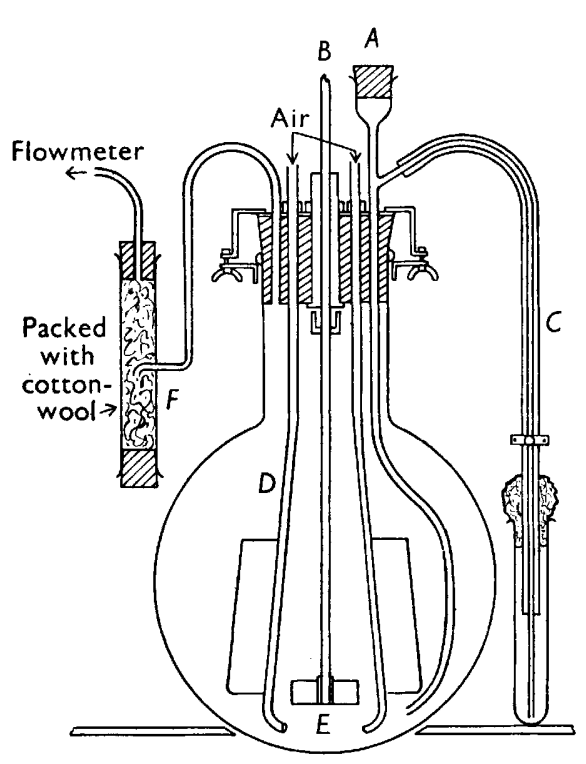

Fig. 1

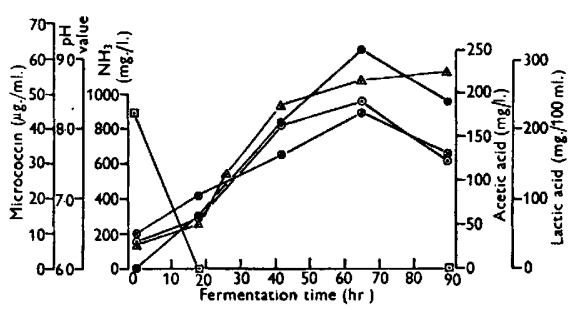

Fig. 2

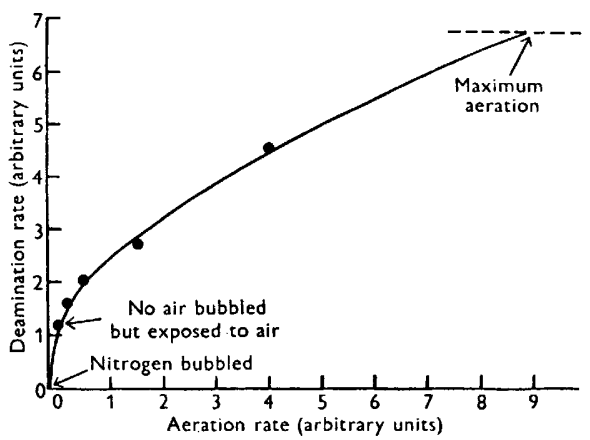

Fig. 3

Fig. 1. Diagram of aerated stirred flask. $A$, inoculator; $B$, stirring shaft; $C$, sampler; $D$, aerator; $E$, stirrer; $F$, outlet air filter.

Fig. 2. Chemical changes in a 70 gal. micrococcin fermentation. Medium: CSL to $1800 \mathrm{mg}$. $\mathrm{N} / \mathrm{l}$; ; Marmite to $1800 \mathrm{mg} . \mathrm{N} / \mathrm{l}$; ; sodium chloride, 0.5 \%. Micrococcin, - pH, $-\triangle-$; ammonia, $-\odot-$; acetic acid, $-\otimes-$; lactic acid, $-\square-$

Fig. 3. Form of curve relating aeration rate to rate of deamination of $\alpha$-alanine by the micrococcus.

Marmite media, while alanine/Marmite medium was considerably better than peptone/Marmite. Lactate also could replace part of the CSL nitrogen but pyruvate could not (Table 2). Glucose was added in experimental fermenta-

Table 2. The influence of alanine, pyruvate, and lactate on micrococcin production

(Basal medium: CSL to provide $1.8 \mathrm{~g} . \mathrm{N} / 1$.; Marmite to provide $1.8 \mathrm{~g} . \mathrm{N} / 1$.; Sodium chloride $0.5 \%$; Sodium sulphate $0.05 \%$; $\mathrm{pH}$ value $=6.5$.)

\section{Medium}

Undiluted

Micrococcin

Diluted 1/2 $\mu \mathrm{g} . / \mathrm{ml}$. (mean)

Diluted $1 / 2+$ alanine to restore to $3 \cdot 6 \mathrm{~g} . \mathrm{N} / 1$.

anine above

Diluted $1 / 2+$ pyruvate to same molarity as alanine above 
tions as an alternative energy source, but it was observed that the low $\mathrm{pH}$ which resulted inhibited micrococcin production. The use of calcium carbonate in such fermentations, was not successful as it did not maintain the $\mathrm{pH}$ high enough (above $\mathrm{pH}$ 6) for maximum production.

Our thanks are due to Prof. Sir Howard Florey, F.R.S., for suggesting this problem and for continued interest in the work; to Miss P. F. Boyd for maintenance of cultures and production of inocula; to Miss N. Smith who was responsible for all assays; to Mr R. C. Codner who suggested modifications of the Kestner vessel; to Mr J. M. Whitmarsh for help in the early stages of this work.

The Engineering Division of The Distillers Co. Ltd., Research and Development Department, kindly assisted in the design and construction of the stirred and aerated fermentation apparatus.

\section{REFERENCES}

Green, C. A. (1946). Penicillin, R.N. production and research. J. R. Naval Sci. Ser. $1,151$.

Heatley, N. G., Kelly, B. K. \& Smith, N. (1952). The assay of micrococcin, an almost insoluble antibiotic. J. gen. Microbiol. 6, 30.

Stephenson, M. (1949). Bacterial Metabolism, 3rd ed. London: Longmans, Green and Co.

Su, T. L. (1948). Micrococcin. An antibacterial substance formed by a strain of micrococcus. Brit. J. exp. Path. 29, 473.

Wise, W. S. (1951). The measurement of the aeration of culture media. J. gen. Microbiol. 5, 167.

EXPLANATION OF PLATE

Plate 1

Fermentation rack showing two frames mounted on one side with stirrers connected up.

(Received 25 May 1951) 
Journal of General Microbiology, Vol. 6, No. $1 \propto 2$

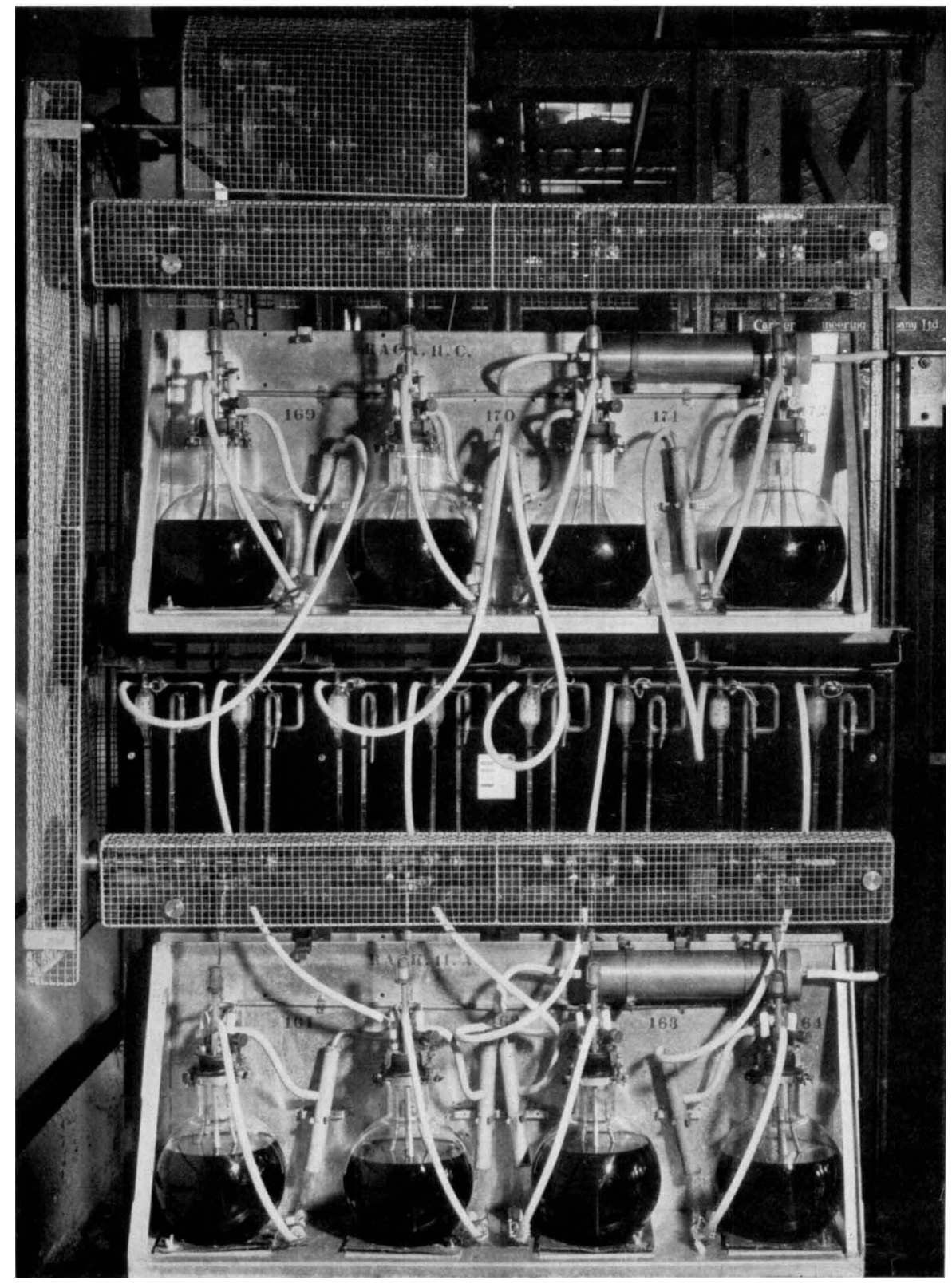

B. K. Kelly, G. A. Milifer \& C. W. Hale-Pronuction of micrococcin. Plate 1 